\title{
A maximum flow formulation of a multi-period open-pit mining problem
}

\author{
Henry Amankwah, Torbjörn Larsson and Björn Textorius
}

\section{Linköping University Post Print}

\section{Tweet}

N.B.: When citing this work, cite the original article.

The original publication is available at www.springerlink.com:

Henry Amankwah, Torbjörn Larsson and Björn Textorius, A maximum flow formulation of a multi-period open-pit mining problem, 2014, Operational Research, (14), 1, 1-10.

http://dx.doi.org/10.1007/s12351-013-0140-7

Copyright: Springer Verlag (Germany)

http://www.springerlink.com/?MUD=MP

Postprint available at: Linköping University Electronic Press

http://urn.kb.se/resolve?urn=urn:nbn:se:liu:diva-106129 


\title{
A maximum flow formulation of a multi-period open-pit mining problem
}

\author{
Henry Amankwah, Torbjörn Larsson†, Björn Textorius ${ }^{\ddagger}$
}

5 January 2014

\begin{abstract}
We consider the problem of finding an optimal mining sequence for an open pit during a number of time periods subject to only spatial and temporal precedence constraints. This problem is of interest because such constraints are generic to any open-pit scheduling problem and, in particular, because it arises as a Lagrangean relaxation of an open-pit scheduling problem. We show that this multi-period open-pit mining problem can be solved as a maximum flow problem in a time-expanded mine graph. Further, the minimum cut in this graph will define an optimal sequence of pits. This result extends a well-known result of J.-C. Picard from 1976 for the open-pit mine design problem, that is, the single-period case, to the case of multiple time periods.
\end{abstract}

Keywords: Open-pit mining, Integer programming, Scheduling, Maximum flow, Maximal closure, Lagrangean relaxation

Mathematics Subject Clasification (2010): 90C10, 90C35, 90C90

\section{Introduction}

Open-pit mining is a surface mining operation whereby ore, or waste, is excavated from the surface of the land, and in so doing a deeper and deeper pit is formed. Before the mining begins, the volume of the ore deposit is usually partitioned into blocks and the value of the ore in each block is

${ }^{*}$ Department of Mathematics and Statistics, University of Cape Coast, Ghana

${ }^{\dagger}$ Department of Mathematics, Linköping University, Sweden

${ }^{\ddagger}$ Department of Mathematics, Linköping University, Sweden 
estimated by using geological information from drill holes. The cost of mining and processing each block is also estimated. A profit can thus be assigned to each block of the mine model, as illustrated in Figure 1.

A fundamental problem in open-pit mine planning is to decide which blocks to mine. This is known as the problem of finding an open-pit mine design, or an ultimate contour for the pit. The only restrictions are spatial precedence relationships, stating that in order to extract any given block, so must all blocks immediately above and within a required wall slope angle. Lerchs and Grossmann (1965) showed that the design problem can be stated as the problem of finding a maximal closure in a mine graph which represents the blocks and the precedence restrictions, as shown in Figure 1 (for a safe slope angle of $45^{\circ}$ ). Their algorithm for finding a maximal closure in the mine graph has over the years been commonly used by the mining industry for the design of open pits.

\begin{tabular}{|c|c|c|c|}
\hline-1 & 3 & 3 & -2 \\
1 & 2 & 3 & 4 \\
\hline & 2 & 8 & \\
5 & 6 & \\
\end{tabular}

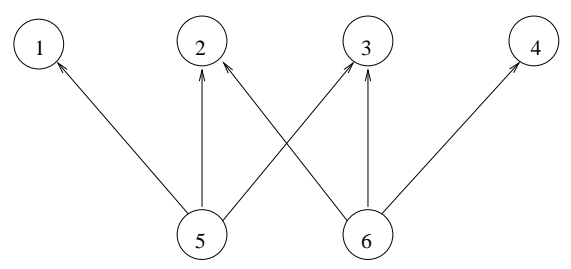

Figure 1: A 2-D block model of a mine with block profit values and its mine graph

The practical siginificance of the open-pit mine design problem makes it an important instance of the maximal closure problem (Picard and Queyranne, 1982). As shown by Picard (1976), the problem of finding a maximal closure in a mine graph can be solved as a maximum flow problem in a network derived from the mine graph, and where a minimum cut determines an optimal pit contour. Later, Hochbaum and Chen (2000) and Hochbaum (2001) developed efficient maximum flow algorithms for the open-pit mining problem.

In reality, the profit of a block depends on when it is mined, for example due to discounting. This fact leads to another crucial issue in open-pit mine planning, namely scheduling. This is the process of deciding how and when to mine the blocks so as to maximize profit (typically the net present value), while obeying the wall slope and precedence constraints, as well as various mining capacity restrictions. Contributions within open-pit mine scheduling, from the view of mathematical optimization, have been given by Gershon (1983), Dagdelen and Johnson (1986), Caccetta and Hill (2003), Ramazan (2007), Rafiee and Asghari (2008), Bley et al. (2010), and Cullenbine et al. (2011), among others. 
We consider a multi-period open-pit mining problem with only spatial and temporal precedence constraints. The latter simply state that once a block has been mined, it shall remain mined. The spatial and temporal precedence constraints are generic to open-pit mine scheduling and the multi-period problem arises as a Lagrangean relaxed open-pit scheduling problem, when capacity restrictions are Lagrangean dualized.

It will be shown that this multi-period open-pit mining problem can be formulated as a maximum flow problem in a time-expanded mine graph, which has a copy of the mine graph for each time period. The expanded graph also contains directed arcs that model the temporal precedence relationships between the corresponding nodes in successive copies of the mine graph; these arcs are analogous to those that model the spatial precedence relationships within each of the mine graphs. This maximum flow formulation extends the result of Picard (1976) to the case of multiple time periods. Figure 2 shows the time-expansion of the mine graph in Figure 1, for the case $T=3$.

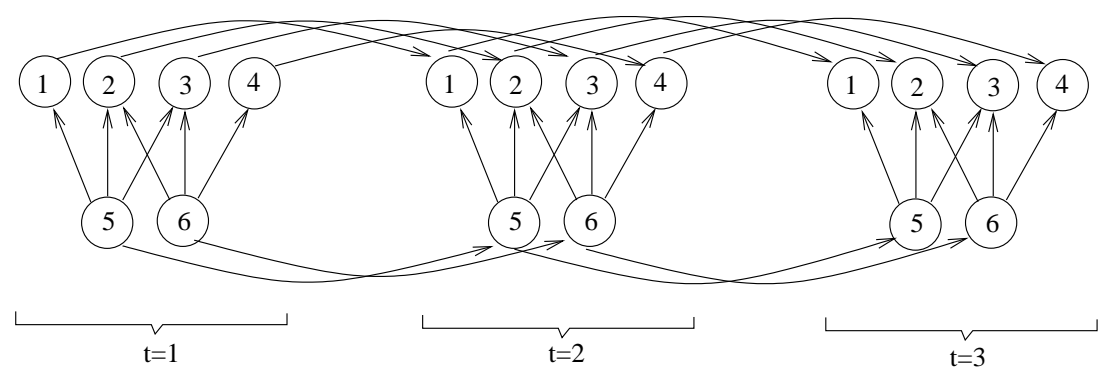

Figure 2: A time-expanded mine graph with three time periods

In Section 2 we give the mathematical model of the problem considered. In Section 3 we present the maximum flow problem in the time-expanded mine graph and show that a minimum cut in this graph defines an optimal solution to the multi-period open-pit mining problem. Section 4 presents a small illustrative example. The last section gives a couple of concluding remarks.

\section{The mathematical model}

The following notation will be used. 
$T=$ number of time periods.

$V=$ set of all blocks that can be mined.

$A=$ set of pairs $(i, j)$ of blocks such that block $j$ is a neighbouring block to $i$ that must be removed before block $i$ can be mined.

$\bar{p}_{i}^{t}=$ contribution to the objective value if block $i$ is mined in time period $t$ or earlier, $i \in V, t=0, \ldots, T$.

Defining the decision variables for all $i \in V$ and $t=1, \ldots, T$ as

$$
x_{i}^{t}=\left\{\begin{array}{l}
1, \text { if block } i \text { is mined in time period } t \text { or earlier } \\
0, \text { otherwise }
\end{array}\right.
$$

the multi-period open-pit mining problem is formulated as

$$
z^{*}=\operatorname{maximize} \sum_{t=0}^{T} \sum_{i \in V} \bar{p}_{i}^{t} x_{i}^{t}
$$

subject to

$$
\begin{aligned}
& x_{i}^{t} \leq x_{j}^{t}, \quad(i, j) \in A, t=1, \ldots, T \\
& x_{i}^{t-1} \leq x_{i}^{t}, \quad t=1, \ldots, T, i \in V \\
& x_{i}^{0}=0, \quad i \in V \\
& x_{i}^{t} \in\{0,1\}, \quad i \in V, t=1, \ldots, T .
\end{aligned}
$$

The first and second sets of constraints are spatial respective temporal precedence restrictions. As shall be shown, an optimal solution to this problem is found by solving a maximum flow problem in the time-expanded mine graph.

\section{The maximum flow formulation}

In order to state the time-expanded maximum flow problem, we introduce the sets of block nodes $V_{t}^{+}=\left\{i \in V \mid \bar{p}_{i}^{t}>0\right\}$ and $V_{t}^{-}=\left\{i \in V \mid \bar{p}_{i}^{t} \leq 0\right\}$, $t=1, \ldots, T$, and further let $\sigma$ and $\tau$ be the source and sink nodes respectively of the network, which includes arcs from the source node to the nodes $i \in V_{t}^{+}$, $t=1, \ldots, T$, and arcs from the nodes $i \in V_{t}^{-}, t=1, \ldots, T$, to the sink node. Letting $J_{i}^{+}=\{j \in V \mid(i, j) \in A\}$ and $J_{i}^{-}=\{j \in V \mid(j, i) \in A\}, i \in V$, the maximum flow problem is as follows.

$$
f^{*}=\operatorname{maximize} \quad f
$$


subject to

$$
\begin{aligned}
& \sum_{t=1}^{T} \sum_{i \in V_{t}^{+}} v_{\sigma i}^{t}=f \\
& \sum_{j \in J_{i}^{+}} y_{i j}^{1}-\sum_{j \in J_{i}^{-}} y_{j i}^{1}+s_{i}^{2}-v_{\sigma i}^{1}=0, \quad i \in V_{1}^{+} \\
& \sum_{j \in J_{i}^{+}} y_{i j}^{t}-\sum_{j \in J_{i}^{-}} y_{j i}^{t}-s_{i}^{t}+s_{i}^{t+1}-v_{\sigma i}^{t}=0, \quad i \in V_{t}^{+}, t=2, \ldots, T-1 \\
& \sum_{j \in J_{i}^{+}} y_{i j}^{T}-\sum_{j \in J_{i}^{-}} y_{j i}^{T}-s_{i}^{T}+v_{\sigma i}^{T}=0, \quad i \in V_{T}^{+} \\
& \sum_{j \in J_{i}^{+}} y_{i j}^{1}-\sum_{j \in J_{i}^{-}} y_{j i}^{1}+s_{i}^{2}+u_{i \tau}^{1}=0, \quad i \in V_{1}^{-} \\
& \sum_{j \in J_{i}^{+}} y_{i j}^{t}-\sum_{j \in J_{i}^{-}} y_{j i}^{t}-s_{i}^{t}+s_{i}^{t+1}+u_{i \tau}^{t}=0, \quad i \in V_{t}^{-}, t=2, \ldots, T-1 \\
& \sum_{j \in J_{i}^{+}} y_{i j}^{T}-\sum_{j \in J_{i}^{-}} y_{j i}^{T}-s_{i}^{T}-u_{i \tau}^{T}=0, \quad i \in V_{T}^{-} \\
& -\sum_{t=1}^{T} \sum_{i \in V_{t}^{-}} u_{i \tau}^{t}=-f \\
& 0 \leq v_{\sigma i}^{t} \leq \bar{p}_{i}^{t}, \quad i \in V_{t}^{+}, t=1, \ldots, T \\
& 0 \leq u_{i \tau}^{t} \leq-\bar{p}_{i}^{t}, \quad i \in V_{t}^{-}, t=1, \ldots, T \\
& s_{i}^{t} \geq 0, \quad i \in V, t=1, \ldots, T \\
& y_{i j}^{t} \geq 0, \quad(i, j) \in A, t=1, \ldots, T
\end{aligned}
$$

Here, $f$ is the total flow, the quantity $y_{i j}^{t}$ is the flow from block node $i$ to block node $j$ in time period $t$, and each $s_{i}^{t}$ corresponds to a forward arc between corresponding block nodes in successive time periods. Further, $v_{\sigma i}^{t}$ is the flow from the source node to block node $i$ in period $t$, while $u_{i \tau}^{t}$ is the flow from block node $i$ in period $t$ to the sink node.

An example of the maximum flow network is given in Figure 3, with 9 blocks and 3 time periods (but with only some of the arcs shown).

Let $(S, \bar{S})$ be a minimum cut in the time-expanded maximum flow network. Then $S=\{\sigma\} \cup S^{1} \cup \ldots \cup S^{T}$ and $\bar{S}=\{\tau\} \cup \bar{S}^{1} \cup \ldots \cup \bar{S}^{T}$, where $\left(S^{t}, \bar{S}^{t}\right)$ is the cut through the mine graph copy for time period $t$. 


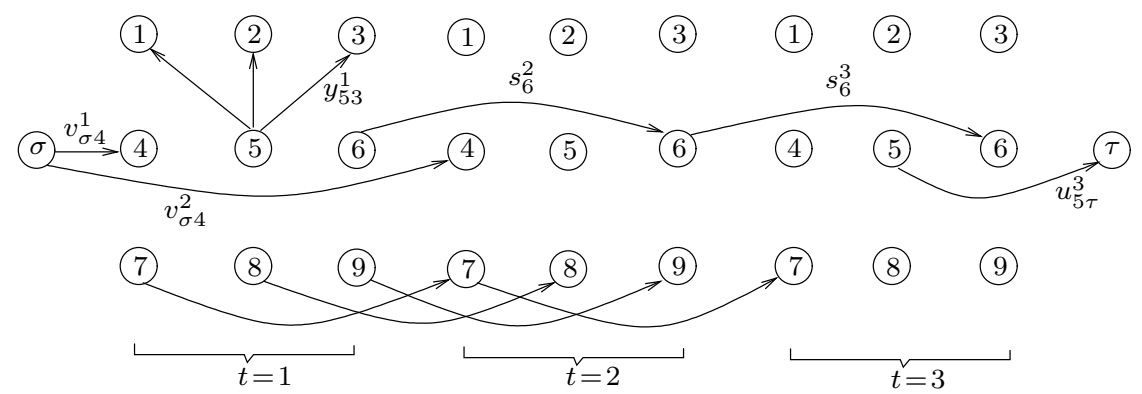

Figure 3: Example of the maximum flow network (with sample arcs)

Theorem 1. An optimal solution to Problem (1) is given by

$$
x_{i}^{t^{*}}=\left\{\begin{array}{l}
1, i \in S^{t} \\
0, i \in \bar{S}^{t}
\end{array}, i \in V, t=1, \ldots, T,\right.
$$

and

$$
z^{*}=\sum_{t=1}^{T} \sum_{i \in V_{t}^{+}} \bar{p}_{i}^{t}-f^{*} .
$$

Proof. We study the linear programming dual of the above maximum flow problem. Let $l_{i}^{t}, i \in V, t=1, \ldots, T$, be the dual variables corresponding to the block nodes in the network, and let $q_{\sigma}$ and $q_{\tau}$ be the respective dual variables associated with the source and the sink. By further introducing $h_{i}^{t}$, $i \in V, t=1, \ldots, T$, as the dual variables for the upper bound constraints, the dual problem becomes

$$
f^{*}=\operatorname{minimize} \sum_{t=1}^{T} \sum_{i \in V_{t}^{+}} \bar{p}_{i}^{t} h_{i}^{t}+\sum_{t=1}^{T} \sum_{i \in V_{t}^{-}}\left(-\bar{p}_{i}^{t}\right) h_{i}^{t}
$$

subject to

$$
\begin{aligned}
& q_{\tau}-q_{\sigma}=1 \\
& q_{\sigma}-l_{i}^{t}+h_{i}^{t} \geq 0, \quad i \in V_{t}^{+}, t=1, \ldots, T \\
& l_{i}^{t}-q_{\tau}+h_{i}^{t} \geq 0, \quad i \in V_{t}^{-}, t=1, \ldots, T \\
& l_{i}^{t}-l_{j}^{t} \geq 0, \quad(i, j) \in A, t=1, \ldots, T \\
& l_{i}^{t-1}-l_{i}^{t} \geq 0, \quad t=2, \ldots, T, \quad i \in V \\
& h_{i}^{t} \geq 0, \quad i \in V, \quad t=1, \ldots, T .
\end{aligned}
$$


An optimal solution to the dual problem is then (e.g., Bazaraa and Jarvis, 1977) given by $q_{\sigma}^{*}=0, q_{\tau}^{*}=1$,

$$
l_{i}^{t^{*}}=\left\{\begin{array}{ll}
0, & i \in S^{t} \\
1, & i \in \bar{S}^{t}
\end{array}, i \in V, t=1, \ldots, T,\right.
$$

and

$$
h_{i}^{t^{*}}=\left\{\begin{array}{ll}
1, & i \in\left(V_{t}^{+} \cap \bar{S}^{t}\right) \cup\left(V_{t}^{-} \cap S^{t}\right) \\
0, & \text { otherwise }
\end{array}, i \in V, t=1, \ldots, T .\right.
$$

Then, for $i \in V_{t}^{+}, t=1, \ldots, T$,

$$
\left.\begin{array}{l}
i \in S^{t} \Longrightarrow h_{i}^{t^{*}}=l_{i}^{*^{*}}=0 \\
i \in \bar{S}^{t} \Longrightarrow h_{i}^{t^{*}}=l_{i}^{t^{*}}=1
\end{array}\right\} \Longrightarrow h_{i}^{t^{*}}=l_{i}^{t^{*}}
$$

and for $i \in V_{t}^{-}, t=1, \ldots, T$,

$$
\left.\begin{array}{l}
i \in \bar{S}^{t} \Longrightarrow h_{i}^{t^{*}}=1-l_{i}^{t^{*}}=0 \\
i \in S^{t} \Longrightarrow h_{i}^{t^{*}}=1-l_{i}^{t^{*}}=1
\end{array}\right\} \Longrightarrow h_{i}^{t^{*}}=1-l_{i}^{t^{*}} .
$$

It then holds that

$$
f^{*}=\operatorname{minimize} \sum_{t=1}^{T} \sum_{i \in V_{t}^{+}} \bar{p}_{i}^{t} h_{i}^{t}+\sum_{t=1}^{T} \sum_{i \in V_{t}^{-}}\left(-\bar{p}_{i}^{t}\right) h_{i}^{t}
$$

subject to the constraints (3)-(8) and to

$$
\begin{aligned}
& q_{\sigma}=0, \\
& q_{\tau}=1, \\
& h_{i}^{t}=l_{i}^{t}, \quad i \in V_{t}^{+}, t=1, \ldots, T \\
& h_{i}^{t}=1-l_{i}^{t}, \quad i \in V_{t}^{-}, t=1, \ldots, T
\end{aligned}
$$

with the optimal solution to Problem (2) still being optimal, since restricting $q_{\sigma}$ and $q_{\tau}$ to their respective optimal values and enforcing the equalities (9) and (10) to hold for any solutions will not affect its optimality.

As is easily verified, constraints (3)-(5) can be removed from Problem (11), since they will always be fulfilled. By further eliminating the variables $q_{\sigma}$, $q_{\tau}$, and $h_{i}^{t}, i \in V, t=1, \ldots, T$, from Problem (11) it is reduced to

$$
f^{*}=\operatorname{minimize} \sum_{t=1}^{T} \sum_{i \in V} \bar{p}_{i}^{t} l_{i}^{t}-\sum_{t=1}^{T} \sum_{i \in V_{t}^{-}} \bar{p}_{i}^{t}
$$


subject to

$$
\begin{aligned}
& l_{i}^{t}-l_{j}^{t} \geq 0, \quad(i, j) \in A, t=1, \ldots, T \\
& l_{i}^{t-1}-l_{i}^{t} \geq 0, \quad t=2, \ldots, T, i \in V \\
& l_{i}^{t} \geq 0, \quad i \in V_{t}^{+}, t=1, \ldots, T . \\
& 1-l_{i}^{t} \geq 0, \quad i \in V_{t}^{-}, t=1, \ldots, T .
\end{aligned}
$$

Now, let $x_{i}^{t}=1-l_{i}^{t}$, for all $i \in V$ and $t=1, \ldots, T$. Then the above problem can be stated as

$$
-f^{*}=\operatorname{maximize} \sum_{t=1}^{T} \sum_{i \in V} \bar{p}_{i}^{t} x_{i}^{t}-\sum_{t=1}^{T} \sum_{i \in V_{t}^{+}} \bar{p}_{i}^{t}
$$

subject to

$$
\begin{array}{lc}
x_{i}^{t} \leq x_{j}^{t}, & (i, j) \in A, t=1, \ldots, T \\
x_{i}^{t-1} \leq x_{i}^{t}, & t=2, \ldots, T, i \in V \\
x_{i}^{t} \leq 1, & i \in V_{t}^{+}, t=1, \ldots, T . \\
x_{i}^{t} \geq 0, & i \in V_{t}^{-}, t=1, \ldots, T,
\end{array}
$$

which is solved by

$$
x_{i}^{t^{*}}=1-l_{i}^{t^{*}}=\left\{\begin{array}{l}
1, i \in S^{t} \\
0, i \in \bar{S}^{t}
\end{array}, i \in V, t=1, \ldots, T .\right.
$$

Since this optimal solution is binary, it follows that it is also an optimal solution to Problem (1). The expression for its optimal value follows directly from the above objective function.

Since the forward arcs corresponding to the variables $s_{i}^{t}$ are not capacitated, it follows that $i \in S^{t}$ whenever $i \in S^{t-1}$, so that $S^{1} \subseteq S^{2} \subseteq \ldots \subseteq S^{T}$ holds. Hence, the sequence of cuts $\left(S^{t}, \bar{S}^{t}\right), t=1, \ldots, T$, define larger and larger pits. The blocks mined precisely in the first time period are those corresponding to the nodes in the set $S^{1}$, while for $t=2, \ldots, T$ it is the blocks corresponding to the nodes in the sets $S^{t} \backslash S^{t-1}$.

\section{An example}

As mentioned in the introduction, the problem under consideration is of interest because it appears when an open-pit mine scheduling problem is 
Lagrangean relaxed. To illustrate this, we consider the following scheduling model, which is a special case of the model considered by Bley et al. (2010).

$$
\operatorname{maximize} \sum_{t=1}^{T} \sum_{i \in V} p_{i}^{t}\left(x_{i}^{t}-x_{i}^{t-1}\right)
$$

subject to

$$
\begin{aligned}
& \sum_{i \in V} b_{i}\left(x_{i}^{t}-x_{i}^{t-1}\right) \leq u^{t}, \quad t=1, \ldots, T \\
& x_{i}^{t} \leq x_{j}^{t}, \quad(i, j) \in A, t=1, \ldots, T \\
& x_{i}^{t-1} \leq x_{i}^{t}, \quad t=1, \ldots, T, \quad i \in V \\
& x_{i}^{0}=0, \quad i \in V \\
& x_{i}^{t} \in\{0,1\}, \quad i \in V, t=1, \ldots, T .
\end{aligned}
$$

The decision variables are defined as above. (Note that the difference $x_{i}^{t}-x_{i}^{t-1}$ takes the value one when block $i$ is mined in exactly time period $t$.) Further, $p_{i}^{t}$ is the profit made from mining block $i$ in time period $t, b_{i}$ is the tonnage of block $i$, and $u^{t}$ is an upper bound on the tonnage mined in time period $t$. Letting $\lambda_{t} \geq 0, t=1, \ldots, T$, be multipliers associated with the constraints on maximal tonnage mined in each time period and Lagrangean relaxing these constraints, we get an instance of Problem (1), with the coefficients in the objective function being the Lagrangean reduced profits

$$
\left\{\begin{array}{l}
\bar{p}_{i}^{0}=-\left(p_{i}^{1}-\lambda_{1} b_{i}\right) \\
\bar{p}_{i}^{t}=\left(p_{i}^{t}-\lambda_{t} b_{i}\right)-\left(p_{i}^{t+1}-\lambda_{t+1} b_{i}\right), t=1, \ldots, T-1, \\
\bar{p}_{i}^{T}=p_{i}^{T}-\lambda_{T} b_{i} .
\end{array}\right.
$$

The reader may note that Problem (1) would also arise as a column generation problem (or, pricing problem) if the linear programming relaxation of Problem (12) is solved by a column generation scheme.

To illustrate the result of the theorem, we consider the block model in Figure 1 and construct an instance of Problem (12) by letting $T=3, u^{t}=2$ for all $t$, and $b_{i}=1$ for all $i$. Further, the profit values are discounted by a factor 0.90 for each time period. To create an instance of Problem (1) we Lagrangean relax the capacity constraints with the multiplier values $\lambda_{1}=$ $0.48, \lambda_{2}=0.18$, and $\lambda_{3}=0$. [These values come from the dual of the linear programming relaxation of Problem (12).] The minimum cut for the timeexpanded maximum flow problem is shown in Figure 4. It indicates that blocks 2 and 3 are mined in the first time period, blocks 4 and 6 in the second, and blocks 1 and 5 in the last. The optimal profit is 12.21 . 


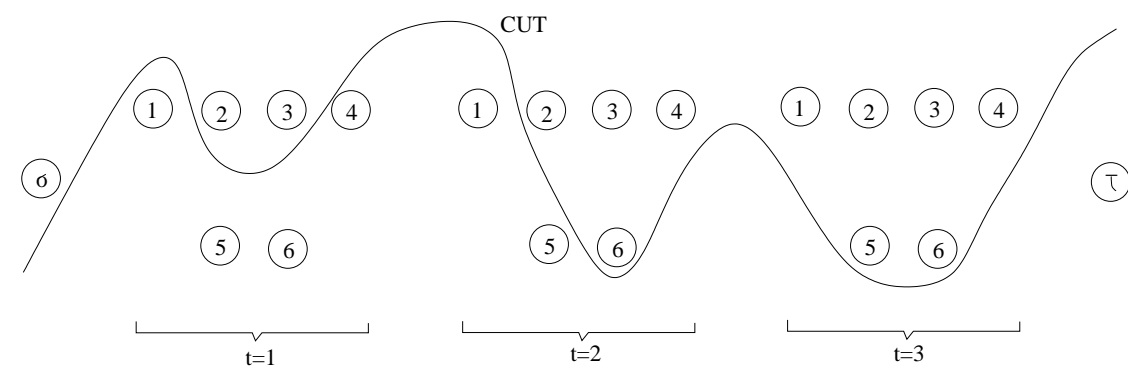

Figure 4: Minimum cut that defines the mining sequence

\section{Conclusion}

We have given a maximum flow formulation of a multi-period open-pit mining problem. It extends the classic maximum flow formulation of Picard (1976) for a single time period by means of a time-expanded network. Picard's derivation is based on a reformulation of the open-pit mine design problem into a quadratic binary program, while our proof of the validity of the timeexpanded maximum flow formulation is based on linear programming duality.

The problem under consideration in this paper arises naturally if all constraints of an open-pit scheduling problem but the spatial and temporal precedence restrictions are Lagrangean dualized, or priced out in a column generation fashion. For any values of the Lagrangean multipliers, the maximum flow solution in the time-expanded network will correspond to a mining schedule that is feasible with respect to both the spatial and temporal precedence restrictions. The Lagrangean multipliers can then be thought of as parameters that shall be tuned such that the capacity restrictions become fulfilled, in an optimal way. Because the prevalence of a duality gap, this strategy cannot however be expected to be sufficient to optimally solve the scheduling problem.

Opportunities for further research are clearly the study of Lagrangean dual and column generation approaches based on the time-expanded maximum flow problem, as a vehicle for solving open-pit mine scheduling problems, heuristically or optimally.

\section{References}

Bazaraa MS, Jarvis JJ (1977) Linear Programming and Network Flows. Wiley, New York, NY

Bley A, Boland N, Fricke C, Froyland G (2010) A strengthened formulation 
and cutting planes for the open pit mine production scheduling problem. Computers and Operations Research 37:1641-1647

Caccetta L, Hill SP (2003) An application of branch and cut to open pit mine scheduling. Journal of Global Optimization 27:349-365

Cullenbine C, Wood RK, Newman A (2011) A sliding time window heuristic for open pit mine block sequencing. Optimization Letters 5:365-377

Dagdelen K, Johnson TB (1986) Optimum open pit mine production scheduling by Lagrangian parameterization. In: Proceedings of the 19th International Symposium on the Application of Computers and Operations Research in the Mineral Industry, AIME. pp 127-142

Gershon ME (1983) Optimal mine production scheduling: evaluation of large scale mathematical programming approaches. International Journal of Mining Engineering 1:315-329

Hochbaum DS (2001) A new-old algorithm for minimum-cut and maximumflow in closure graphs. Networks 37:171-193

Hochbaum DS, Chen A (2000) Performance analysis and best implementations of old and new algorithms for the open-pit mining problem. Operations Research 48:894-914

Lerchs H, Grossmann IF (1965) Optimum design of open-pit mines. Transactions, Canadian Institute of Mining and Metallurgy LXVIII:17-24

Picard J-C (1976) Maximal closure of a graph and applications to combinatorial problems. Management Science 22:1268-1272

Picard J-C, Queyranne M (1982) Selected applications of minimum cuts in networks. INFOR 20:394-422

Rafiee V, Asghari O (2008) A heuristic traditional MIP solving approach for long term production scheduling in open pit mine. Journal of Applied Sciences 8:4512-4522

Ramazan S (2007) The new fundamental tree algorithm for production scheduling of open pit mines. European Journal of Operational Research $177: 1153-1166$ 